\title{
Efeitos de uma pandemia numa unidade de cuidados de saúde primários e sua população: um estudo retrospetivo
}

Pedro Alexandre Vieira', Paulo Jorge Barros' ${ }^{1}$ Tânia Caseiro' ${ }^{1}$ Natalina Rodrigues², João Arcanjo³

\section{RESUMO}

Objetivos: A COVID-19 é a primeira pandemia severa do mundo moderno, tendo já colapsado diversos sistemas de saúde, obrigando-os a adaptar-se. Este artigo pretende analisar a atividade médica numa unidade de cuidados de saúde primários durante o primeiro pico da pandemia, de modo a avaliar os impactos desta no acompanhamento clínico da população.

Métodos: Estudo retrospetivo que analisou os dados de todas as consultas efetuadas numa unidade de cuidados primários entre 1 de março e 31 de julho de 2020 e em período homólogo de 2019 e 2018.

Resultados: Em 2020 registou-se um aumento de consultas quando comparado com igual período de 2019 e 2018 (14.871, 14.326 e 12.996 , respetivamente), sendo a maioria não presencial, ao contrário de anos anteriores (71,6\% em $2020,42,6 \%$ em 2019 e 35,2\% em 2018). Verificou-se também uma diminuição da maioria das consultas de vigilância e de rotina (2.562 em 2020, 3.067 em 2019 e 2.975 em 2018) e um aumento das consultas por patologia psiquiátrica, destacando-se a ansiedade (362 em 2020, 252 em 2019 e 238 em 2018).

Conclusões: Apesar da pandemia, o número total de consultas aumentou em 2020, embora à custa de consultas não presenciais e com uma diferente prevalência de diagnósticos. Ocorreram menos consultas de vigilância e aumentaram as consultas por patologia psiquiátrica, possivelmente decorrentes do impacto socioeconómico da pandemia. Trata-se da primeira casuística da atividade médica de cuidados primários registada durante a primeira vaga da pandemia em Portugal.

Palavras-chave: COVID-19; Pandemia; Cuidados de saúde primários; Centros de saúde.

\section{INTRODUÇÃO}

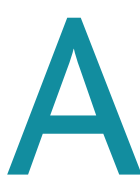
doença COVID-19 é uma síndroma respiratória aguda, potencialmente severa, causada pelo coronavírus 2 (SARS-CoV-2). Foi inicialmente detetada em Wuhan, na China, em dezembro de 2019, e rapidamente se propagou por todo o mundo, tendo sido declarada como pandemia pela Organização Mundial da Saúde (OMS) no dia 11 de

1. Médico Interno de Medicina Geral e Familiar. USF Mondego, Centro de Saúde de S. Martinho do Bispo. Coimbra, Portugal.

2. Médica Assistente de Medicina Geral e Familiar. USF Mondego, Centro de Saúde de S. Martinho do Bispo. Coimbra, Portugal.

3. Médico Assistente Graduado de Medicina Geral e Familiar. USF Mondego, Centro de Saúde de S. Martinho do Bispo. Coimbra, Portugal. março de 2020. ${ }^{1}$ Tem tido consequências devastadoras nos sistemas de saúde, na economia e na população de todo o globo, tendo já infetado, à data da elaboração deste artigo, mais de 110.000.000 de pessoas e sendo causa de morte de mais de $2.490 .000 .^{2}$ Trata-se da primeira pandemia severa do mundo moderno, tendo já sido capaz de levar ao colapso diversos sistemas de saúde de países desenvolvidos. ${ }^{3-4}$

Em Portugal, o primeiro caso de COVID-19 foi confirmado no dia 2 de março de 2020 e, a partir daí, a pandemia já infetou mais de 790.000 pessoas e vitimou mais de $16.000 .{ }^{5}$ Além destes, muitos mais foram também indiretamente afetados pela pandemia, não só a 
nível da saúde, como também ao nível económico e social. $^{6}$

Os serviços de saúde tiveram de se adaptar a esta nova realidade, tendo-se privilegiado as consultas não presenciais com o intuito de diminuir o risco de exposição ao vírus dos profissionais e dos utentes. Neste âmbito, a 13 de março de 2020 foram suspensas as atividades presenciais das unidades de cuidados primários consideradas não prioritárias, como os rastreios oncológicos e as consultas de vigilância de saúde, mantendo-se apenas o atendimento de utentes com patologia aguda, descompensação de patologia crónica, rastreio metabólico do recém-nascido, vacinação e outros considerados inadiáveis. ${ }^{7-8}$

Assim, com este artigo, pretende-se avaliar o impacto da pandemia de COVID-19 na população e na atividade assistencial dos cuidados de saúde primários em Portugal.

Para este fim, foi caracterizada a atividade assistencial médica de uma Unidade de Cuidados de Saúde Primários localizada no Centro de Portugal entre os meses de março e julho de 2020 e foi comparada com período homólogo de 2019 e 2018. Foram também analisados os diagnósticos das consultas efetuadas durante este período e comparados com igual período de 2019 e 2018, de modo a avaliar os impactos da pandemia na saúde da população.

\section{MÉTODOS}

\section{Desenho do estudo e local}

Efetuou-se um estudo retrospetivo, observacional e descritivo na Unidade de Saúde Familiar (USF) Mondego, uma unidade de cuidados de saúde primários localizada no Centro de Saúde de S. Martinho do Bispo, na região de Coimbra. Esta Unidade encontra-se integrada no Agrupamento de Centros de Saúde (ACeS) do Baixo Mondego.

Foram incluídos dados de todas as consultas ocorridas entre 1 de março e 31 de julho de 2020 e em período homólogo de 2019 e 2018, recolhidos retrospetivamente em agosto de 2020, na Unidade de Saúde, recorrendo ao MIM@UF, plataforma que agrega os dados relativos às consultas efetuadas na Unidade e devidamente registadas na plataforma SClínico®, o sistema de informação utilizado na Unidade.

Este intervalo de tempo foi selecionado por contemplar o período no qual se verificou o maior impac- to da pandemia. A retoma da carteira básica de serviços das Unidades ocorreu praticamente em pleno a partir de agosto de 2020.

O estudo foi aprovado pela Comissão de Ética para a Saúde da Administração Regional de Saúde (ARS) do Centro e foi realizado de acordo com os princípios estabelecidos na Declaração de Helsínquia.

\section{Colheita de dados e variáveis}

Os dados foram recolhidos através da plataforma MIM@UF para uma base de dados em Excel®. As variáveis selecionadas incluíram: género, idade, número de consultas de cada utente no período selecionado (presenciais, não presenciais e total), frequência de cada diagnóstico ICPC-2 (International Classification of Primary Care 2), colocado no campo «A» do registo clínico SOAP das consultas do período selecionado.

Todas as consultas realizadas na Unidade foram registadas no sistema de informação SClínico®. Em todas as consultas, presenciais ou não, este sistema exige o preenchimento do campo «A» do SOAP com, pelo menos, um diagnóstico codificado de acordo com a classificação ICPC-2.

Os dados recolhidos dizem respeito apenas à USF Mondego.

\section{Análise estatística}

Os dados foram recolhidos e processados com recurso ao Microsoft Office Excel 2016® e ao IBM SPSS Statistics v. 26.

Aplicou-se o teste Qui-quadrado de Pearson para avaliar a associação estatística entre as variáveis tipo de consulta (presencial e não presencial) e ano (2018, 2019 e 2020). Nas consultas não presenciais foram incluídas as realizadas através do e-mail, a consulta telefónica, renovação de receituário, elaboração de relatórios clínicos, análise e interpretação de exames complementares de diagnóstico e de informação proveniente de outros prestadores de cuidados de saúde, como cartas de alta do serviço de urgência ou de internamento hospitalar.

Definiu-se o nível de significância estatística a 5\%.

\section{RESULTADOS}

\section{Consultas efetuadas}

Entre os meses de março e julho de 2018, 2019 e 2020 foram realizadas, respetivamente, 12.996, $14.326 \mathrm{e}$ 
TABELA 1. Casuística das consultas efetuadas na USF Mondego entre os meses de março e julho de 2020, 2019 e 2018

\begin{tabular}{l|c|c|c|c} 
Ano & $\mathbf{2 0 2 0}$ & $\mathbf{2 0 1 9}$ & $\mathbf{2 0 1 8}$ & $\boldsymbol{P}$ \\
\hline $\mathrm{N}^{\circ}$ de consultas presenciais & 4.230 & 8.217 & 8.415 & $<0,00001$ \\
[n(\%)] & $(28,4 \%)$ & $(57,4 \%)$ & $(64,8 \%)$ & \\
\hline $\mathrm{N}^{\circ}$ consultas não presenciais & 10.641 & 6.109 & 4.581 & $<0,00001$ \\
{$[\mathrm{n}(\%)]$} & $(71,6 \%)$ & $(42,6 \%)$ & $(35,2 \%)$ & \\
\hline $\mathrm{N}^{\circ}$ de consultas total & 14.871 & 14.326 & 12.996 & \\
[n(\%)] & $(100 \%)$ & $(100 \%)$ & $(100 \%)$ & \\
\hline
\end{tabular}

Valor Qui-quadrado de Pearson $\left(X^{2}\right)=4200,4$

TABELA 2. Cobertura assistencial das consultas efetuadas na USF Mondego entre os meses de março e julho de 2020, 2019 e 2018

\begin{tabular}{l|c|c|c} 
Ano & 2020 & 2019 & 2018 \\
\hline $\mathrm{N}^{\circ}$ de utentes em consulta presencial & 3.260 & 5.160 & 5.074 \\
{$[\mathrm{n}(\%)]$} & $(32,6 \%)$ & $(53,1 \%)$ & $(53,6 \%)$ \\
\hline $\mathrm{N}^{\circ}$ de utentes em consulta não & 4.958 & 3.634 & 2.812 \\
presencial [n(\%)] & $(49,6 \%)$ & $(37,4 \%)$ & $(29,7 \%)$ \\
\hline $\mathrm{N}^{\circ}$ de utentes com pelo menos um dos & 6.238 & 6.596 & 6.127 \\
tipos de consulta [n(\%)] & $(62,3 \%)$ & $(67,9 \%)$ & $(64,7 \%)$ \\
\hline Utentes inscritos na USF (julho do ano) & 10.006 & 9.709 & 9.475 \\
[n(\%)] & $(100 \%)$ & $(100 \%)$ & $(100 \%)$ \\
\hline
\end{tabular}

Legenda: USF = Unidade de Saúde Familiar.

14.871 consultas na Unidade estudada. Na Tabela 1 encontra-se resumida a casuística das consultas deste período de tempo.

$\mathrm{Na}$ Tabela 1 verifica-se que no período estudado de 2020 se registou uma clara predominância de consultas não presenciais, correspondendo a 71,6\% (10.641) do total de consultas, enquanto nos anos de 2019 e 2018 esta percentagem foi sempre inferior a $43 \%$. Contudo, em 2020, constata-se que o total de consultas efetuadas no intervalo de tempo estudado foi superior ao período homólogo dos dois anos anteriores, com um aumento de 3,8\% ( +545 consultas) relativamente a 2019 e de $14,4 \%$ ( +1.875 consultas) relativamente a 2018 .

Uma vez que o valor $p$ é inferior ao nível de significância $(0,05)$, verifica-se que existe variação estatisticamente significativa entre a proporção de consultas presenciais e não presenciais e os anos estudados (2018, 2019 e 2020).
Na Tabela 2 constata-se que a proporção de utentes inscritos na Unidade que teve acesso a consulta médica presencial em $2020(32,6 \%)$ foi inferior quando comparada com período homólogo de 2019 (53,1\%) e 2018 (53,6\%). Contudo, a proporção de utentes com consulta não presencial aumentou comparativamente a anos anteriores. Analisando a proporção de utentes que teve acesso a pelo menos um dos tipos de consulta verifica-se que esta percentagem, em 2020, desceu para 62,3\% em comparação com os anos anteriores. Ou seja, apesar do número total de consultas da Unidade ter aumentado em 2020, registou-se uma menor proporção dos utentes inscritos na Unidade que teve acesso a consulta.

A distribuição do número de consultas por utente frequentador encontra-se resumida na Tabela 3 .

Na Tabela 3 verifica-se que, em relação às consultas presenciais, entre março e julho de 2020, comparativamente a igual período de 2019 e 2018, houve um menor número de utentes com acesso a mais do que uma consulta, existindo uma maior percentagem $(79,2 \%)$ de utentes utilizadores com acesso a apenas uma consulta entre março e julho. Também o número máximo de consultas por utente utilizador no período estudado foi de nove; em 2018 e 2019 este valor foi de 12.

Já nas consultas não presenciais, nas quais se incluiu a consulta telefónica ou por $e$-mail, a renovação de receituário ou a orientação de exames complementares ou informação de outros prestadores de saúde, esta relação inverte-se; no período estudado de 2020 a maioria dos utentes utilizadores teve mais do que uma consulta com o médico, ao contrário dos anos anteriores. Também o máximo de consultas não presenciais por utente utilizador foi maior em 2020, com um máximo de $17 \mathrm{em}$ dois utentes; este valor foi de 13 em 2019 e de 15 em 2018.

Quanto ao total de consultas, presenciais e não presenciais, por utente frequentador, apurou-se que a percentagem de utentes utilizadores com mais do que uma consulta médica é superior em 2020. Também o máximo de consultas por utente utilizador é superior em 


\begin{tabular}{|c|c|c|c|c|c|c|c|c|c|}
\hline \multirow{2}{*}{$\begin{array}{l}\mathrm{N} .{ }^{\circ} \text { de consultas } \\
\text { por utente } \\
\text { utilizador }\end{array}$} & \multicolumn{3}{|c|}{ Consultas presenciais } & \multicolumn{3}{|c|}{ Consultas não presenciais } & \multicolumn{3}{|c|}{$\begin{array}{c}\text { Consultas presenciais e não } \\
\text { presenciais }\end{array}$} \\
\hline & 2020 & 2019 & 2018 & 2020 & 2019 & 2018 & 2020 & 2019 & 2018 \\
\hline $\begin{array}{l}1 \\
{[n(\%)]}\end{array}$ & $\begin{array}{c}2.583 \\
(79,2 \%)\end{array}$ & $\begin{array}{c}3.345 \\
(64,8 \%) \\
\end{array}$ & $\begin{array}{c}3.136 \\
(62,3 \%) \\
\end{array}$ & $\begin{array}{c}2.447 \\
(49,4 \%)\end{array}$ & $\begin{array}{c}2.249 \\
(61,9 \%)\end{array}$ & $\begin{array}{c}1.794 \\
(63,8 \%) \\
\end{array}$ & $\begin{array}{c}2.797 \\
(44,8 \%)\end{array}$ & $\begin{array}{c}3.164 \\
(48,0 \%) \\
\end{array}$ & $\begin{array}{c}2.996 \\
(48,9 \%) \\
\end{array}$ \\
\hline$[n(\%)]$ & $\begin{array}{c}473 \\
(14,5 \%)\end{array}$ & $\begin{array}{c}1.097 \\
(21,3 \%)\end{array}$ & $\begin{array}{c}1.131 \\
(22,3 \%)\end{array}$ & $\begin{array}{c}1.163 \\
(23,5 \%)\end{array}$ & $\begin{array}{c}778 \\
(21,4 \%)\end{array}$ & $\begin{array}{c}613 \\
(21,8 \%)\end{array}$ & $\begin{array}{c}1.476 \\
(23,7 \%)\end{array}$ & $\begin{array}{c}1.526 \\
(23,1 \%)\end{array}$ & $\begin{array}{c}1.464 \\
(23,9 \%)\end{array}$ \\
\hline $\begin{array}{l}3 \\
{[n(\%)]}\end{array}$ & $\begin{array}{c}146 \\
(4,5 \%)\end{array}$ & $\begin{array}{c}416 \\
(8,1 \%)\end{array}$ & $\begin{array}{c}444 \\
(8,8 \%)\end{array}$ & $\begin{array}{c}595 \\
(12,0 \%)\end{array}$ & $\begin{array}{c}335 \\
(9,2 \%)\end{array}$ & $\begin{array}{c}233 \\
(8,3 \%)\end{array}$ & $\begin{array}{c}753 \\
(12,1 \%)\end{array}$ & $\begin{array}{c}850 \\
(12,9 \%)\end{array}$ & $\begin{array}{c}741 \\
(12,1 \%)\end{array}$ \\
\hline $\begin{array}{l}4 \\
{[n(\%)]}\end{array}$ & $\begin{array}{c}39 \\
(1,2 \%)\end{array}$ & $\begin{array}{c}171 \\
(3,3 \%)\end{array}$ & $\begin{array}{c}167 \\
(3,3 \%)\end{array}$ & $\begin{array}{c}338 \\
(6,8 \%)\end{array}$ & $\begin{array}{c}162 \\
(4,5 \%)\end{array}$ & $\begin{array}{c}99 \\
(3,5 \%)\end{array}$ & $\begin{array}{c}474 \\
(7,6 \%)\end{array}$ & $\begin{array}{c}468 \\
(7,1 \%)\end{array}$ & $\begin{array}{c}430 \\
(7,0 \%)\end{array}$ \\
\hline $\begin{array}{l}5 \\
{[n(\%)]}\end{array}$ & $\begin{array}{c}13 \\
(0,4 \%)\end{array}$ & $\begin{array}{c}79 \\
(1,5 \%)\end{array}$ & $\begin{array}{c}88 \\
(1,7 \%)\end{array}$ & $\begin{array}{c}175 \\
(3,5 \%)\end{array}$ & $\begin{array}{c}61 \\
(1,7 \%)\end{array}$ & $\begin{array}{c}35 \\
(1,2 \%)\end{array}$ & $\begin{array}{c}279 \\
(4,5 \%)\end{array}$ & $\begin{array}{c}272 \\
(4,1 \%)\end{array}$ & $\begin{array}{c}213 \\
(3,5 \%)\end{array}$ \\
\hline $\begin{array}{l}6 \\
{[n(\%)]}\end{array}$ & $\begin{array}{c}3 \\
(0,1 \%)\end{array}$ & $\begin{array}{c}30 \\
(0,6 \%)\end{array}$ & $\begin{array}{c}52 \\
(1,0 \%)\end{array}$ & $\begin{array}{c}104 \\
(2,1 \%)\end{array}$ & $\begin{array}{c}24 \\
(0,7 \%)\end{array}$ & $\begin{array}{c}17 \\
(0,6 \%)\end{array}$ & $\begin{array}{c}188 \\
(3,0 \%)\end{array}$ & $\begin{array}{c}125 \\
(1,9 \%)\end{array}$ & $\begin{array}{c}124 \\
(2,0 \%)\end{array}$ \\
\hline $\begin{array}{l}7 \\
{[n(\%)]}\end{array}$ & $\begin{array}{c}1 \\
(0,0 \%)\end{array}$ & $\begin{array}{c}14 \\
(0,3 \%)\end{array}$ & $\begin{array}{c}12 \\
(0,2 \%)\end{array}$ & $\begin{array}{c}47 \\
(0,9 \%)\end{array}$ & $\begin{array}{c}11 \\
(0,3 \%)\end{array}$ & $\begin{array}{c}8 \\
(0,3 \%)\end{array}$ & $\begin{array}{c}96 \\
(1,5 \%)\end{array}$ & $\begin{array}{c}85 \\
(1,3 \%)\end{array}$ & $\begin{array}{c}76 \\
(1,2 \%)\end{array}$ \\
\hline $\begin{array}{l}8 \\
{[n(\%)]}\end{array}$ & $\begin{array}{c}1 \\
(0,0 \%)\end{array}$ & $\begin{array}{c}3 \\
(0,1 \%)\end{array}$ & $\begin{array}{c}8 \\
(0,2 \%)\end{array}$ & $\begin{array}{c}27 \\
(0,5 \%)\end{array}$ & $\begin{array}{c}9 \\
(0,2 \%)\end{array}$ & $\begin{array}{c}3 \\
(0,1 \%)\end{array}$ & $\begin{array}{c}56 \\
(0,9 \%)\end{array}$ & $\begin{array}{c}50 \\
(0,8 \%)\end{array}$ & $\begin{array}{c}39 \\
(0,6 \%)\end{array}$ \\
\hline $\begin{array}{l}9 \\
{[n(\%)]}\end{array}$ & $\begin{array}{c}1 \\
(0,0 \%)\end{array}$ & $\begin{array}{c}3 \\
(0,1 \%)\end{array}$ & $\begin{array}{c}3 \\
(0,1 \%)\end{array}$ & $\begin{array}{c}16 \\
(0,3 \%)\end{array}$ & $\begin{array}{c}1 \\
(0,0 \%)\end{array}$ & $\begin{array}{c}2 \\
(0,1 \%)\end{array}$ & $\begin{array}{c}42 \\
(0,7 \%)\end{array}$ & $\begin{array}{c}21 \\
(0,3 \%)\end{array}$ & $\begin{array}{c}15 \\
(0,2 \%)\end{array}$ \\
\hline $\begin{array}{l}10 \\
{[n(\%)]}\end{array}$ & 0 & $\begin{array}{c}1 \\
(0,0 \%)\end{array}$ & $\begin{array}{c}4 \\
(0,1 \%)\end{array}$ & $\begin{array}{c}12 \\
(0,2 \%)\end{array}$ & $\begin{array}{c}2 \\
(0,1 \%)\end{array}$ & $\begin{array}{c}5 \\
(0,2 \%)\end{array}$ & $\begin{array}{c}17 \\
(0,3 \%)\end{array}$ & $\begin{array}{c}14 \\
(0,2 \%)\end{array}$ & $\begin{array}{c}9 \\
(0,1 \%)\end{array}$ \\
\hline $\begin{array}{l}11 \\
{[n(\%)]}\end{array}$ & 0 & 0 & $\begin{array}{c}1 \\
(0,0 \%)\end{array}$ & $\begin{array}{c}14 \\
(0,3 \%)\end{array}$ & $\begin{array}{c}1 \\
(0,0 \%)\end{array}$ & 0 & $\begin{array}{c}21 \\
(0,3 \%)\end{array}$ & $\begin{array}{c}11 \\
(0,2 \%)\end{array}$ & $\begin{array}{c}8 \\
(0,1 \%)\end{array}$ \\
\hline $\begin{array}{l}12 \\
{[n(\%)]}\end{array}$ & 0 & $\begin{array}{c}1 \\
(0,0 \%)\end{array}$ & $\begin{array}{c}1 \\
(0,0 \%)\end{array}$ & $\begin{array}{c}9 \\
(0,2 \%)\end{array}$ & 0 & $\begin{array}{c}1 \\
(0,0 \%)\end{array}$ & $\begin{array}{c}13 \\
(0,2 \%)\end{array}$ & $\begin{array}{c}6 \\
(0,1 \%)\end{array}$ & $\begin{array}{c}4 \\
(0,1 \%)\end{array}$ \\
\hline $\begin{array}{l}13 \\
{[n(\%)]}\end{array}$ & 0 & 0 & 0 & $\begin{array}{c}5 \\
(0,1 \%) \\
\end{array}$ & $\begin{array}{c}1 \\
(0,0 \%)\end{array}$ & 0 & $\begin{array}{c}11 \\
(0,2 \%)\end{array}$ & 0 & $\begin{array}{c}4 \\
(0,1 \%)\end{array}$ \\
\hline $\begin{array}{l}14 \\
{[n(\%)]}\end{array}$ & 0 & 0 & 0 & 0 & 0 & $\begin{array}{c}1 \\
(0,0 \%)\end{array}$ & $\begin{array}{c}3 \\
(0,0 \%)\end{array}$ & $\begin{array}{c}2 \\
(0,0 \%)\end{array}$ & 0 \\
\hline $\begin{array}{l}15 \\
{[n(\%)]}\end{array}$ & 0 & 0 & 0 & $\begin{array}{c}2 \\
(0,0 \%)\end{array}$ & 0 & $\begin{array}{c}1 \\
(0,0 \%)\end{array}$ & $\begin{array}{c}3 \\
(0,0 \%)\end{array}$ & 0 & $\begin{array}{c}2 \\
(0,0 \%)\end{array}$ \\
\hline $\begin{array}{l}16 \\
{[n(\%)]}\end{array}$ & 0 & 0 & 0 & $\begin{array}{c}2 \\
(0,0 \%)\end{array}$ & 0 & 0 & $\begin{array}{c}2 \\
(0,0 \%)\end{array}$ & $\begin{array}{c}1 \\
(0,0 \%)\end{array}$ & $\begin{array}{c}1 \\
(0,0 \%)\end{array}$ \\
\hline $\begin{array}{l}17 \\
{[n(\%)]}\end{array}$ & 0 & 0 & 0 & $\begin{array}{c}2 \\
(0,0 \%)\end{array}$ & 0 & 0 & $\begin{array}{c}4 \\
(0,1 \%)\end{array}$ & $\begin{array}{c}1 \\
(0,0 \%)\end{array}$ & $\begin{array}{c}1 \\
(0,0 \%)\end{array}$ \\
\hline $\begin{array}{l}18 \\
{[n(\%)]}\end{array}$ & 0 & 0 & 0 & 0 & 0 & 0 & $\begin{array}{c}2 \\
(0,0 \%)\end{array}$ & 0 & 0 \\
\hline
\end{tabular}


TABELA 3. Número de consultas por utente utilizador (continuação)

\begin{tabular}{|c|c|c|c|c|c|c|c|c|c|}
\hline \multirow{2}{*}{$\begin{array}{l}\text { N. }{ }^{\circ} \text { de consultas } \\
\text { por utente } \\
\text { utilizador }\end{array}$} & \multicolumn{3}{|c|}{ Consultas presenciais } & \multicolumn{3}{|c|}{ Consultas não presenciais } & \multicolumn{3}{|c|}{$\begin{array}{c}\text { Consultas presenciais e não } \\
\text { presenciais }\end{array}$} \\
\hline & 2020 & 2019 & 2018 & 2020 & 2019 & 2018 & 2020 & 2019 & 2018 \\
\hline $\begin{array}{l}23 \\
{[n(\%)]}\end{array}$ & 0 & 0 & 0 & 0 & 0 & 0 & $\begin{array}{c}1 \\
(0,0 \%)\end{array}$ & 0 & 0 \\
\hline $\begin{array}{l}\text { Total de utentes } \\
\text { utilizadores } \\
{[n(\%)]}\end{array}$ & $\begin{array}{c}3.260 \\
(100 \%)\end{array}$ & $\begin{array}{c}5.160 \\
(100 \%)\end{array}$ & $\begin{array}{c}5.074 \\
(100 \%)\end{array}$ & $\begin{array}{c}4.958 \\
(100 \%)\end{array}$ & $\begin{array}{c}3.634 \\
(100 \%)\end{array}$ & $\begin{array}{c}2.812 \\
(100 \%)\end{array}$ & $\begin{array}{c}6.238 \\
(100 \%)\end{array}$ & $\begin{array}{c}6.596 \\
(100 \%)\end{array}$ & $\begin{array}{c}6.127 \\
(100 \%)\end{array}$ \\
\hline $\begin{array}{l}\text { Média de } \\
\text { consultas por } \\
\text { utente utilizador }\end{array}$ & 1,30 & 1,59 & 1,66 & 2,15 & 1,68 & 1,63 & 2,38 & 2,17 & 2,12 \\
\hline
\end{tabular}

\section{TABELA 4. Distribuição das consultas efetuadas por sexo}

\begin{tabular}{l|c|c|c|c|c|c|c|c|c}
\multirow{3}{*}{ Sexo } & \multicolumn{4}{|c|}{ Consultas presenciais } & \multicolumn{3}{c|}{ Consultas não presenciais } & \multicolumn{3}{c}{$\begin{array}{c}\text { Consultas presenciais e não } \\
\text { presenciais }\end{array}$} \\
\cline { 2 - 10 } & 2020 & 2019 & $\mathbf{2 0 1 8}$ & 2020 & 2019 & $\mathbf{2 0 1 8}$ & 2020 & 2019 & 2018 \\
\hline Feminino & 2.498 & 5.009 & 5.385 & 6.533 & 3.913 & 2.788 & 9.031 & 8.922 & 8.173 \\
{$[n(\%)]$} & $(59,1 \%)$ & $(61,0 \%)$ & $(64,0 \%)$ & $(61,4 \%)$ & $(64,1 \%)$ & $(60,9 \%)$ & $(60,7 \%)$ & $(62,3 \%)$ & $(62,9 \%)$ \\
\hline Masculino & 1.732 & 3.208 & 3.030 & 4.108 & 2.196 & 1.793 & 5.840 & 5.404 & 4.823 \\
{$[n(\%)]$} & $(40,9 \%)$ & $(39,0 \%)$ & $(36,0 \%)$ & $(38,6 \%)$ & $(35,9 \%)$ & $(39,1 \%)$ & $(39,3 \%)$ & $(37,7 \%)$ & $(37,1 \%)$ \\
\hline Total & 4.230 & 8.217 & 8.415 & 10.641 & 6.109 & 4.581 & 14.871 & 14.326 & 12.996 \\
{$[n(\%)]$} & $(100 \%)$ & $(100 \%)$ & $(100 \%)$ & $(100 \%)$ & $(100 \%)$ & $(100 \%)$ & $(100 \%)$ & $(100 \%)$ & $(100 \%)$ \\
\hline
\end{tabular}

2020, com um utente com 23 consultas, valor inferior ao máximo de 17 de um utente em 2018 e 2019.

\section{Características demográficas dos utentes}

Quanto às características demográficas dos utentes observados em consulta, as mesmas encontram-se resumidas nas Tabelas 4 e 5 .

A análise da Tabela 4 constata uma distribuição semelhante das consultas por sexo dos utentes frequentadores dos vários tipos de consulta, realizadas entre março e julho de 2020, 2019 e 2018. Existe, em todos os anos, um predomínio de consultas no sexo feminino.

Já na Tabela 5 observa-se que a proporção de consultas presenciais em 2020, quando comparada com o ano anterior, reduziu-se entre $42,0 \%$ a $53,9 \%$ em todas as faixas etárias. Por outro lado, ao nível das consultas não pre- senciais verificou-se uma tendência inversa, com um aumento superior a 50\% em todas as faixas etárias, à exceção da faixa etária dos [10-18[ anos, em que este aumento, em 2020, ficou em 20,5\% quando comparado com o ano anterior. Saliente-se que, na faixa etária dos [18-40[ anos, a proporção de consultas não presenciais por utente deste grupo mais do que duplicou, com um aumento de 118,0\% em 2020 face a 2019. Contudo, ao analisar a evolução do total de consultas (presenciais e não presenciais) constatam-se valores semelhantes entre os anos analisados, salientando-se um aumento em 2020, quando comparado com 2019 , de $12,9 \%$ na faixa etária $\geq 80$ anos e uma diminuição de 22,1\% no grupo dos [10-18[ anos e de 15,9\% no grupo dos [0-10[anos. Já na caracterização da média de idades dos utentes observados em consulta verifica-se que é semelhante entre os diversos anos. 


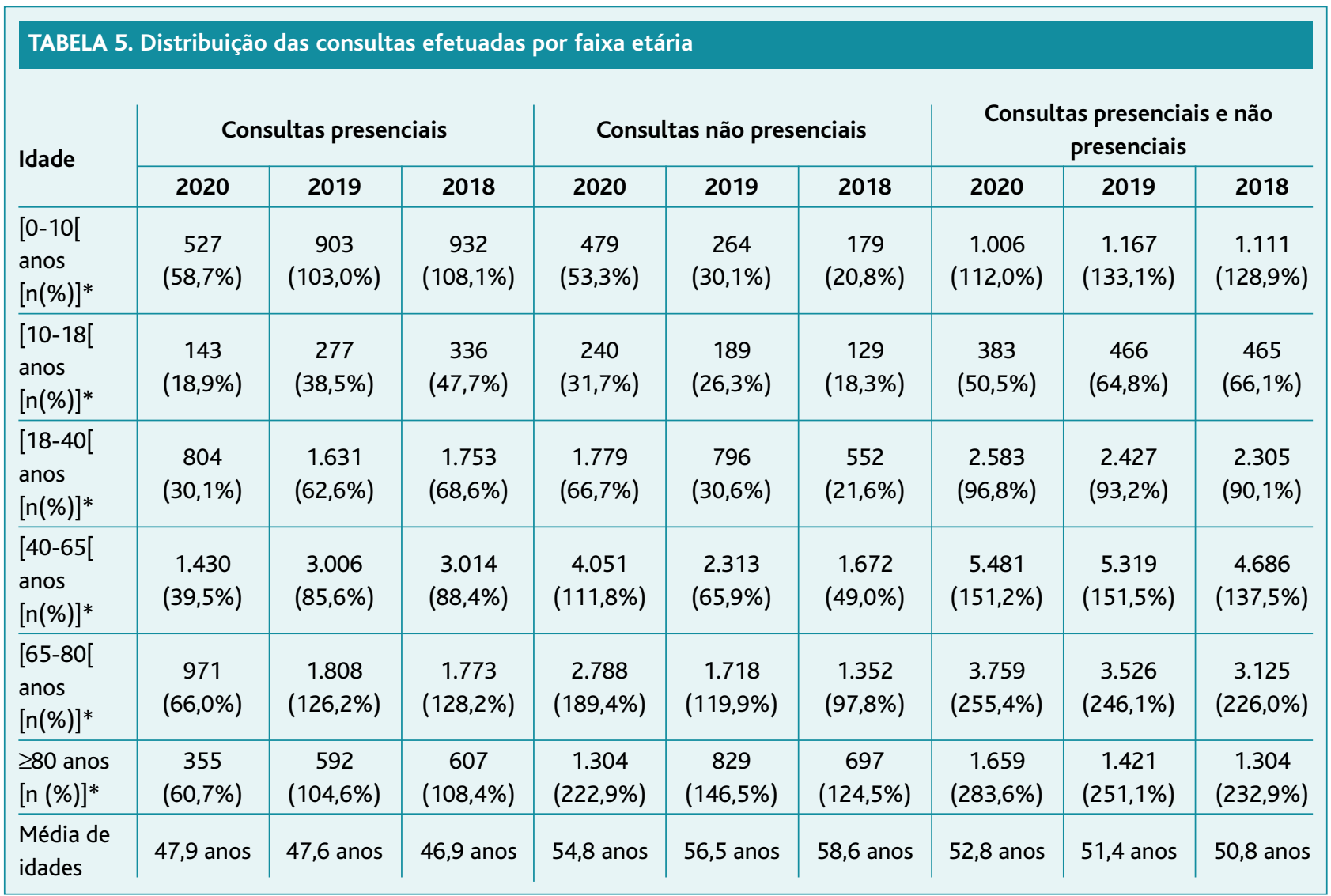

* Percentagem relativa ao total de utentes de cada faixa etária inscritos na Unidade em cada ano:

2020: [0-10[ anos: 898 utentes; [10-18[ anos: 758 utentes; [18-40[ anos: 2.669 utentes; [40-65[ anos: 3.624 utentes; [65-80[ anos: 1.472 utentes; $\geq 80$ anos: 585

2019: [0-10[ anos: 877 utentes; [10-18[ anos: 719 utentes; [18-40[ anos: 2.604 utentes; [40-65[ anos: 3.510 utentes; [65-80[ anos: 1.433 utentes; $\geq 80$ anos: 566

2018: [0-10[ anos: 862 utentes; [10-18[ anos: 704 utentes; [18-40[ anos: 2.557 utentes; [40-65[ anos: 3.409 utentes; [65-80[ anos: 1.383 utentes; $\geq 80$ anos: 560

\section{Diagnósticos das consultas}

Os 15 diagnósticos mais frequentemente codificados em consulta (presencial e não presencial) encontram-se resumidos na Figura 1.

Quanto aos 15 principais diagnósticos das consultas realizadas entre março e julho verifica-se que é muito semelhante nos três anos em estudo. Contudo, há uma redução marcada de consultas com o código diagnóstico ICPC-2 «A98 - Medicina Preventiva/de Acompanhamento geral» no campo A do registo clínico SOAP, geralmente utilizado nas consultas de vigilância de saúde infantil e juvenil, rastreio e consulta de rotina. Constata-se, de igual modo, uma discreta diminuição das consultas com a codificação diagnóstica de hipertensão arterial. Já a frequência da codificação do diagnóstico de diabetes mellitus teve uma tendência inversa, com um aumento no número de consultas com esta codificação, apesar de em frequência relativa ter ultrapassado os valores de 2019, mas não os de 2018.

Relativamente à patologia psiquiátrica, apurou-se um aumento das consultas com a codificação de perturbação do sono e ansiedade em 2020 face aos anos anteriores. Assim, em 2020, quando comparado com 2019, o número de consultas com a codificação de perturbação do sono aumentou de 421 para 464 consultas, correspondendo a um aumento de 10,2\%. Já para a 


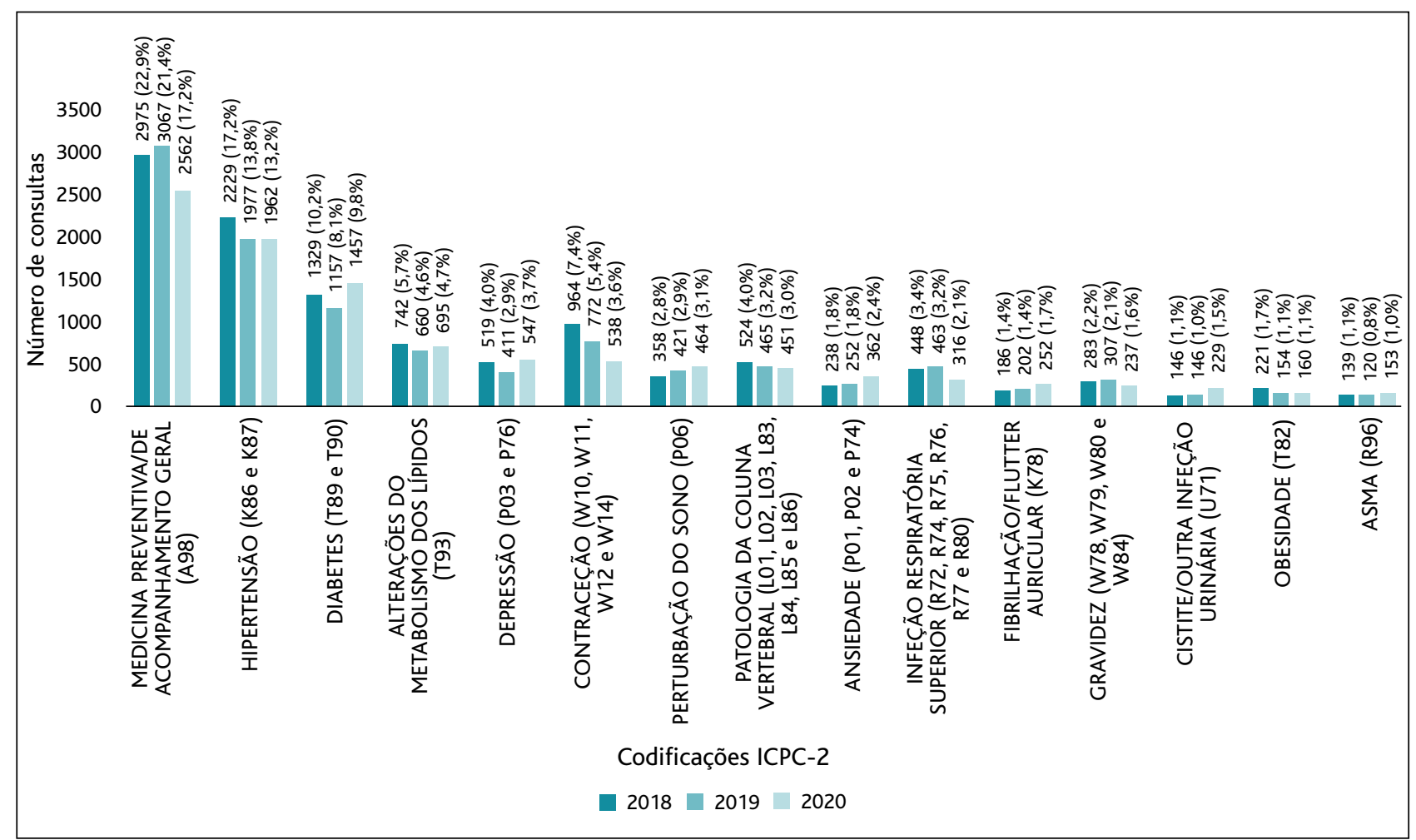

Figura 1. 15 diagnósticos mais frequentemente codificados nas consultas de março a julho de 2020 em comparação com igual período de 2019 e 2018.

Nota 1: A percentagem refere-se à percentagem de consultas do período em estudo com a respetiva codificação. Total de consultas: $2020: 14.871$ (100\%); 2019: 14.326 (100\%); 2018: 12.996 (100\%).

Nota 2: Entre parêntesis encontram-se os códigos ICPC-2 (International Classification of Primary Care 2) incluídos em cada secção: Ansiedade: inclui P01 - Sensação de ansiedade/nervosismo/tensão, P02 - Reação aguda ao stress e P74 - Distúrbio ansioso/estado de ansiedade; Contraceção: inclui W10 - Contraceção pós-coital, W11 - Contraceção oral, W12 - Contraceção intrauterina e W14 - Contraceção, outros; Depressão: inclui P03 Sensação de depressão e P76 - Perturbação depressiva; Diabetes: inclui T89 - Diabetes insulinodependente e T90 - Diabetes não insulinodependente; Gravidez: inclui W78 - Gravidez, W79 - Gravidez não desejada, W80 - Gravidez ectópica e W84 - Gravidez de alto risco; Hipertensão: inclui K86 - Hipertensão sem complicações e K87 - Hipertensão com complicações; Infeção respiratória superior: inclui R72 - Infeção estreptocócica da orofaringe, R74 - Infeção aguda do aparelho respiratório superior, R75 - Sinusite crónica/aguda, R76 - Amigdalite aguda, R77 - Laringite/traqueíte aguda e R80 - gripe; Patologia da coluna vertebral: inclui L01 - Sinal/sintoma do pescoço, L02 - Sinal/sintoma da região dorsal, L03 - Sinal/sintoma da região lombar, L83 - Síndroma da coluna cervical, L84 - Síndroma da coluna sem irradiação de dor, L85 - Deformação adquirida da coluna e L86 Síndroma da coluna com irradiação de dores.

ansiedade este aumento foi mais expressivo, de $43,7 \%$, correspondendo a um aumento de 252 para 362 consultas no mesmo período.

Na codificação de depressão, os resultados encontrados nos três anos analisados apresentam uma variação inconsistente. De facto, o número absoluto de consultas com a codificação diagnóstica de depressão foi superior em 2020 face a 2019 e 2018. Contudo, quando analisada a frequência relativa tendo em conta a percentagem total de consultas, este valor, apesar de superior ao en- contrado em 2019, é inferior ao encontrado em 2018.

Quanto às consultas com os códigos de contraceção, onde se incluem as consultas de planeamento familiar e renovação de método contracetivo, registou-se uma diminuição destas em 2020 face a 2019 e 2018.

Saliente-se também que ocorreu uma diminuição das consultas por sintomas da coluna vertebral e por infeção respiratória superior, enquanto a codificação de «U71 - Cistite/outra infeção urinária» sofreu um aumento face a anos anteriores. 


\section{DISCUSSÃO}

\section{Consultas efetuadas}

Durante os meses de março e julho de 2020 assistiu-se a um aumento acentuado do número de consultas não presenciais, comparativamente com anos anteriores, apesar do decréscimo marcado do número de consultas presenciais. Contudo, foi o ano com maior número total de consultas, com um aumento de $3,8 \%$ ( $n=545)$ comparativamente ao período homólogo do ano anterior. Assim, verifica-se que a atividade assistencial médica presencial que era efetuada em anos anteriores pode ter sido largamente substituída e compensada pela consulta não presencial, concretizada através do $e$-mail e consulta telefónica.

De facto, no período de tempo analisado em 2020, a Unidade relatada neste artigo sofreu reformulações de estruturação e funcionamento interno, de acordo com as orientações da ARS Centro. ${ }^{7-8}$ Assim, foi alargado o período de atendimento telefónico e $e$-mail para todo o período de funcionamento da Unidade, numa tentativa de colmatar a diminuição das consultas presenciais. Atente-se que em todas as solicitações de consulta médica no período estudado o utente era previamente contactado por via telefónica ou e-mail, cabendo ao médico decidir se a situação exposta podia ser resolvida por este meio ou se seria necessário agendar observação presencial.

Quanto aos dados relativos ao número de consultas médicas efetuadas em Portugal nos cuidados de saúde primários, ${ }^{9}$ durante os meses de março a julho, foram realizadas 12.315 .334 consultas em 2018, $12.319 .847 \mathrm{em}$ 2019 e 11.634.029 em 2020. Quanto à proporção de consultas presenciais nos intervalos temporais estudados, em 2018 foi de 69,9\% ( $n=8.614 .179$ ), em 2019 de 69,1\% ( $n=8.507 .513)$ e em 2020 de $34,6 \%$ ( $n=4.022 .175)$. Assim, como no presente estudo, também a nível nacional se verificou um aumento marcado da proporção de consultas não presenciais em 2020, comparativamente ao período homólogo de 2019 e 2018. Porém, e contrariamente aos dados encontrados no presente trabalho, em Portugal, o número total de consultas efetuadas no período analisado em 2020 foi inferior a anos anteriores, com uma redução de 5,6\% ( $n=685.818$ consultas) relativamente ao período homólogo de $2019 .{ }^{9}$

As consultas à distância, antes da pandemia de COVID-19, já existiam, embora com muito menor procura, sendo geralmente privilegiada a consulta presencial. Contudo, os profissionais de saúde foram capazes de se reinventar e em muitos países também foi descrito o aumento marcado destas consultas, recorrendo ao telefone, $e$-mail ou consulta virtual, incluindo a avaliação de doentes suspeitos de infeção por COVID-19. ${ }^{10}$ Países como o Canadá, ${ }^{11}$ Reino Unido, ${ }^{12-13}$ Finlândia e Países Baixos receberam orientações para oferecer consultas remotas sempre que possível, com um aumento substancial deste tipo de consultas também nos cuidados primários. ${ }^{10,13-15}$ Estima-se que as consultas à distância tenham correspondido a mais de $50 \%$ das consultas realizadas durante a primeira vaga da pandemia nos Países Baixos. ${ }^{10}$

Quanto à proporção de utentes inscritos na Unidade em estudo que teve acesso a pelo menos um tipo de consulta, presencial ou não presencial, verifica-se que aumentou a cobertura por consultas não presenciais apesar do decréscimo da cobertura por consultas presenciais. No total menos 358 utentes acederam a pelo menos um dos tipos de consulta no período estudado de 2020, em comparação com o ano anterior, com a taxa de cobertura a diminuir de 67,9\% em 2019 para $62,3 \%$ em 2020. Portanto, por esta análise, apesar de ter ocorrido um maior número total de consultas em 2020, estas abrangeram uma menor percentagem dos utentes inscritos na Unidade. Pode-se concluir que cada utente utilizador, em média, teve maior número de consultas entre março e julho de 2020 do que em período homólogo de 2019 e 2018. Este facto é confirmado pelo aumento da média de consultas não presenciais e totais por utente utilizador no período analisado em 2020, quando comparado com igual período de 2019 e 2018. As consultas não presenciais, em 2020, ultrapassaram pela primeira vez a média de duas consultas por utente utilizador desta tipologia de consulta. Saliente-se também que um doente contactou com o seu médico 23 vezes e outro 18 vezes no período analisado, sendo que, em 2018 e 2019, no mesmo período, o máximo do total de consultas por utente não ultrapassou as 17 consultas. Até ao momento da elaboração deste artigo não existiam publicados outros trabalhos a analisar a variação do número de consultas por utente utilizador durante a pandemia de COVID-19.

\section{Diagnósticos das consultas}

A pandemia de COVID-19, além dos milhares de 
infetados e mortos que causou, também interferiu indiretamente na restante população, tendo várias consultas presenciais sido adiadas, o que levou a um menor diagnóstico, monitorização e seguimento de outras patologias. De facto, um estudo promovido pela Escola Nacional de Saúde Pública durante a primeira vaga da pandemia em Portugal mostrou que, nos cuidados de saúde primários, $56 \%$ das consultas dos utentes inquiridos foram realizadas, $27 \%$ presencialmente e $29 \%$ à distância, tendo $21 \%$ sido desmarcadas pelo utente e $23 \%$ pela unidade de saúde. ${ }^{16}$

Neste âmbito, e seguindo as orientações emanadas pela ARS Centro, ${ }^{7-8}$ todas as consultas presenciais não essenciais da Unidade relatada neste artigo foram adiadas ou substituídas por consulta não presencial, o que justifica a diminuição marcada das codificações ICPC-2 «A98 - Medicina preventiva/de acompanhamento geral», também utilizada nas consultas de vigilância de saúde infantil e juvenil e rastreio. De referir que a maioria destas foi adiada, à exceção das consultas de saúde infantil nas idades inferiores a 24 meses e naquelas em que era necessária a atualização do Plano Nacional de Vacinação, como a consulta dos cinco anos. ${ }^{17}$ Contudo, o receio da utilização dos serviços de saúde pode ter reduzido a vacinação das crianças, ${ }^{18} \mathrm{com}$ risco de futuros surtos de doenças evitáveis pela vacinação. ${ }^{19}$ Em Inglaterra, em abril de 2020 , três semanas após a introdução das medidas de confinamento, o número de vacinas antisarampo parotidite e rubéola (VASPR) administradas diminuiu $19,8 \%$, comparativamente com igual período de 2019. ${ }^{20}$ Também no Michigan, nos Estados Unidos, o número de vacinas administradas entre janeiro e abril de 2020 , sofreu uma redução de $21,5 \%$ na faixa etária 0-18 anos e de 15,5\% na faixa 0-2 anos, quando comparado com período homólogo de 2018 e 2019. ${ }^{21}$ Em Singapura, ${ }^{18}$ o número de vacinas administradas durante a primeira vaga da pandemia sofreu uma redução entre $25,6 \%$ a $73,6 \%$ para a vacinaVASPR, de $0,4 \%$ a $10,3 \%$ para a vacina pentavalente contra o tétano, difteria, tosse convulsa, poliomielite e Haemophilus influenza, e de $8,0 \%$ a $67,8 \%$ para a vacina antipneumocócica, quando comparado com período homólogo de 2019. Consequentemente, constata-se uma redução da imunidade de grupo para $74-84 \%$ entre os 12 e 24 meses de idade, abaixo dos $95 \%$ de cobertura necessários para proteção contra o sarampo. ${ }^{18}$
Relativamente às consultas com o diagnóstico de contraceção, mais frequentemente utilizado nas consultas de planeamento familiar, realização do rastreio do cancro do colo uterino e renovação do método contracetivo, sofreram uma drástica diminuição face a 2019 e 2018, o que também se explica pelo adiamento das consultas deste tipo e pela suspensão dos rastreios durante este período de tempo na Região Centro do país. ${ }^{7}$ O mesmo se verificou nas consultas com o código de gravidez, que sofreram uma redução nos meses de março a julho de 2020, face a 2019 e 2018. Isto não significa necessariamente um menor número de gestações, mas antes um maior espaçamento temporal entre as consultas de vigilância, privilegiando apenas as consideradas essenciais ou em que ocorresse alguma intercorrência. ${ }^{7}$ De facto, a suspensão dos rastreios oncológicos pode ter levado a que muitos cancros em estádio inicial tenham ficado por diagnosticar. ${ }^{22}$ Em Espanha, durante o estado de emergência, houve uma redução de $48 \%$ de novos diagnósticos de cancro colorretal entre março e junho de 2020, comparativamente com período homólogo de 2019, devido à suspensão dos programas de rastreio, por restrições na realização de exames endoscópicos e pelo receio dos doentes em recorrer aos hospitais. ${ }^{23}$ Nos Países Baixos também a suspensão dos programas nacionais de rastreio e a menor procura de cuidados de saúde levou a que, entre março e abril de 2020, ocorresse uma redução dos diagnósticos de cancro de mama e cancro colorretal. ${ }^{24}$

Também as codificações de hipertensão arterial, utilizadas nas consultas de vigilância desta patologia, sofreram uma discreta diminuição na Unidade analisada neste trabalho. Contudo, a diminuição não foi maior porque, sempre que possível, solicitava-se que o utente realizasse a medição da tensão arterial no domicílio e enviasse os valores obtidos por meios não presenciais. Posteriormente, a medicação era ajustada pela mesma via, se necessário. Já as codificações de diabetes mellitus, utilizadas também no âmbito da vigilância desta patologia, não sofreram quebra significativa, tendo até aumentado o seu número absoluto face a anos anteriores. Esta situação deve-se ao facto de se ter realizado a respetiva consulta por via não presencial, com envio por parte do utente de registos de peso e tensão arterial do domicílio e doseamentos de hemoglobina glicada. 
Um estudo retrospetivo realizado na Catalunha, em Espanha, avaliou o efeito da pandemia e das medidas de confinamento nos indicadores de desempenho de 288 unidades de cuidados primários, responsáveis pela saúde de cerca de seis milhões de pessoas. ${ }^{25}$ Comparando abril de 2020 com igual período de 2019 destacou-se uma redução de $4,1 \%$ no indicador relativo ao rastreio do pé diabético, $3,4 \%$ no controlo do colesterol LDL, 2,6\% no controlo da pressão arterial, 2,2\% na realização do rastreio de retinopatia diabética e 1,9\% no controlo da hemoglobina glicada dos diabéticos. Durante o confinamento também foi descrita uma redução alarmante na procura de cuidados de saúde por síndroma coronário agudo na Europa e nos Estados Unidos, sendo que os que procuravam cuidados médicos experienciavam uma taxa de mortalidade mais elevada do que previamente à pandemia. ${ }^{19,26-28}$ Em Itália foi registada uma diminuição de $48,4 \%$ das admissões hospitalares por enfarte agudo do miocárdio em 2020, comparativamente ao período homólogo de 2019. ${ }^{27}$ Já em Inglaterra, esta diminuição de admissões pelo mesmo motivo foi de $40 \%$ em março de 2020 , comparativamente a igual período de 2019. ${ }^{28}$

Quanto à codificação de patologia psiquiátrica, tanto na síndroma depressiva como na ansiedade e perturbação do sono, registou-se um aumento em 2020 na Unidade estudada relativamente ao período homólogo de 2019 e 2018. Contudo, nas codificações por perturbação depressiva, apesar do aumento do número absoluto de consultas em 2020 com esta codificação diagnóstica, comparativamente com os anos anteriores, em frequência relativa a percentagem deste código superou os dados de 2019, mas não os de 2018, apesar de ter ficado próximo. Acredita-se que este número possa estar subestimado, uma vez que o receio do vírus pode ter levado a que muitos utentes evitassem os cuidados de saúde pelo risco de contágio. ${ }^{29}$

A análise das consequências de epidemias prévias, como a síndroma respiratória aguda grave (SARS - $s e-$ vere acute respiratory syndrome), o vírus Ébola e a gripe H1N1, indica uma alta prevalência de doença mental entre pacientes, casos suspeitos, contactos de risco, profissionais de saúde da "linha da frente" e nos indivíduos submetidos a isolamento ou medidas de quarentena. ${ }^{30-32}$ Problemas relacionados com a saúde mental prevalentes incluíram depressão, ansiedade, stress psicológico, burnout, ataques de pânico, perturbação de stress pós-traumático, insónia, ${ }^{30-31}$ abuso de substâncias, ${ }^{6}$ irritabilidade e mesmo suicídio. ${ }^{6,32}$ De facto, o confinamento domiciliário é uma experiência desagradável, em que a separação dos entes queridos, perda de liberdade, incerteza sobre o estado da doença, aborrecimento e as dificuldades financeiras e laborais podem ser fatores desencadeantes de perturbações da saúde mental. ${ }^{6,32-33} \mathrm{Em}$ Portugal, a estimativa da prevalência de doença mental relacionada com o COVID-19 é ainda limitada. Contudo, um estudo promovido pela Escola Nacional de Saúde Pública, que decorreu durante o primeiro pico da pandemia em Portugal, aplicou 160.157 questionários e demonstrou que $80,7 \%$ dos inquiridos referiu sentir-se "agitado, ansioso, em baixo ou triste", sendo que $16,5 \%$ referia sentir-se assim quase todos os dias e $9,7 \%$ todos os dias. ${ }^{34}$ Foi também realizado um inquérito a 4.126 profissionais de saúde entre os dias 2 e 10 de abril de 2020 , dos quais $76,7 \%$ reportaram níveis moderados a altos de fadiga e $68,8 \%$ apresentaram níveis de ansiedade elevados. ${ }^{35}$ Ainda um outro estudo, que consistiu na aplicação de quatro escalas validadas a 550 indivíduos, 52,5\% residentes no Brasil e 47,5\% em Portugal, entre 27 de maio e 8 de julho de 2020, revelou uma prevalência de ansiedade de 71,3\% (ligeira em $43,1 \%$ ), de depressão de $24,7 \%$ e sofrendo de ambas as patologias 23,8\% dos inquiridos..$^{33}$ As prevalências encontradas são mais elevadas que as previstas para Portugal para 2020 na ausência da pandemia, onde se previa uma prevalência da perturbação de ansiedade de $21 \%$ e da perturbação depressiva de $17 \%{ }^{36}$

Noutros países foram também reportados aumentos da patologia psiquiátrica com a pandemia de COVID-19. ${ }^{6}$ Em Itália, um estudo com 18.147 indivíduos avaliou os efeitos da pandemia e do confinamento na saúde mental da população ao fim de três a quatro semanas. ${ }^{37}$ Foram reportados níveis elevados de stress pós-traumático em $37,1 \%$ dos inquiridos, depressão severa em $17,3 \%$, ansiedade severa em $20,8 \%$, insónia severa em 7,3\% e sintomas compatíveis com reação de ajustamento em $23,0 \%$. Na China, um ensaio com 1.210 indivíduos encontrou prevalências de ansiedade moderada a severa de $28,8 \%$, de depressão moderada a severa de $16,5 \%$ e 53,8\% dos inquiridos classificou o impacto psicológico da pandemia como moderado ou severo. ${ }^{38}$ Outro estudo na China, com 52.730 indivíduos, relatou 
impacto psicológico negativo da pandemia de COVID-19 em $35 \%$ dos inquiridos, principalmente mulheres e jovens adultos. ${ }^{39}$ Já um estudo multicêntrico com 1.047 indivíduos de diversos continentes (40\% de África, 36\% da Ásia, 21\% da Europa e 3\% de outros) avaliou as consequências psicológicas do confinamento domiciliário durante a pandemia de COVID-19, em comparação com o período antes do mesmo. ${ }^{40}$ Foi reportado um aumento de $12,9 \%$ da sensação de baixo bem-estar mental e um aumento de $10 \%$ dos sintomas depressivos.

Por outro lado, na Unidade relatada neste artigo também se objetivou uma diminuição das consultas com a codificação de patologia da coluna lombar e de infeção respiratória superior. Pode justificar-se, não necessariamente pela menor incidência destas patologias, mas antes pela menor procura dos cuidados de saúde por parte dos utentes com estas patologias pelo receio de contágio por COVID-19. Contudo, também não se pode excluir que o confinamento, a redução da atividade laboral e o maior distanciamento social possam também ter reduzido a incidência destas patologias. ${ }^{15}$ Até ao momento da elaboração deste artigo havia ainda poucos trabalhos publicados acerca da redução da incidência destas patologias com o confinamento. Contudo, os dados encontrados pelo presente trabalho estão de acordo com um estudo realizado na Finlândia durante a primeira vaga da pandemia, em que se registou uma redução de $16 \%$ nas idas ao serviço de urgência hospitalar, com uma diminuição de $31 \%$ das admissões por dor da coluna ou dos membros e de $28 \%$ nas admissões por doenças infeciosas. ${ }^{15}$

Por fim, evidenciou-se também um aumento das consultas com a codificação «U71 - Cistite/outra infeção urinária». À data da presente investigação não foram encontrados outros artigos publicados sobre a incidência da cistite nos cuidados primários durante a pandemia. Contudo, um estudo realizado na Finlândia mostrou uma redução de $17 \%$ das visitas ao serviço de urgência hospitalar por doenças do foro urinário, sugerindo que estas possam ter sido orientadas fora do serviço de urgência hospitalar. ${ }^{15}$ Portanto, pode ter havido um desvio destes utentes para outro nível de cuidados, como para os cuidados primários.

Até à redação deste artigo não existiam trabalhos publicados sobre os diagnósticos das consultas de cuidados primários durante a pandemia de COVID-19. Con- tudo, está descrita uma diminuição da procura de cuidados de saúde pelo receio de contágio, nomeadamente pelos doentes não COVID-19. ${ }^{26}$

\section{Limitações}

Como limitações dos resultados obtidos poder-se-á destacar o facto de se tratar de uma análise retrospetiva da população de apenas uma unidade de saúde localizada na região de Coimbra, no centro do Portugal, fora das zonas mais afetadas diretamente pela pandemia de COVID-19.

Por outro lado, a maior preocupação em registar todas as consultas nos sistemas de informação, a suspensão das taxas moderadoras em todas as consultas dos cuidados de saúde primários desde abril de 2020 e o aumento progressivo do número de utentes inscritos na unidade de saúde em estudo também podem justificar, em parte, os resultados encontrados, nomeadamente relativos ao aumento do número de consultas ocorridas entre março e julho de 2020, quando comparado com igual período de 2019 e 2018.

Contudo, apesar do aumento verificado, o número de consultas pode estar subestimado. Efetivamente, alguns contactos com o médico de família (contacto telefónico ou e-mail para esclarecimento de dúvidas ou o utente que quer apenas um rápido esclarecimento de uma dúvida com o médico) não foram por vezes registados no sistema de informação da Unidade, não tendo, por isso, sido contabilizados no total de consultas realizadas.

Também as variações encontradas nas codificações dos diagnósticos das consultas podem estar sujeitas a viés. Estas podem não ser devidas diretamente a uma diminuição ou aumento da incidência de determinada patologia, mas eventualmente a uma codificação menos detalhada no período de tempo analisado, pela sobrecarga e pressão a que os profissionais estiveram sujeitos.

Também as variações encontradas nas codificações de patologias crónicas podem estar subestimadas. $\mathrm{Na}$ verdade, a anterior consulta de vigilância presencial pode ter sido substituída apenas por renovação "não presencial" de receituário, sem reavaliação do utente, sendo essa consulta também codificada com o diagnóstico da patologia a que se destina a medicação prescrita; por exemplo, o utente portador de diabetes 
mellitus, que não tenha a sua consulta de vigilância, necessitará de renovação do receituário prescrito, podendo ter na mesma uma consulta não presencial registada para este efeito com o código diagnóstico de diabetes, sem ter sido avaliado pelo médico.

Por fim, é de notar que os resultados encontrados não podem ser extrapolados para todo o país, uma vez que a Região Centro, onde se localiza a unidade de saúde descrita no estudo, não foi das mais afetadas pela pandemia no período temporal analisado. Contudo, permite realçar que, mesmo as populações menos afetadas diretamente pela pandemia, tiveram uma alteração no seu acompanhamento clínico e uma distribuição diferente nos diagnósticos das consultas efetuadas.

\section{CONCLUSÃO}

Este artigo permite avaliar o impacto da pandemia na atividade assistencial de uma unidade de cuidados de saúde primários, concluindo que apesar do adiamento das consultas presenciais não essenciais, entre março e julho de 2020, se registou um maior número total de consultas quando comparado com período homólogo de 2019 e 2018. A percentagem de consultas não presenciais correspondeu à maioria das consultas, ao contrário de anos anteriores. Em termos de distribuição demográfica dos utentes avaliados em consulta por sexo e faixa etária, não houve diferenças em 2020 comparativamente com 2019 e 2018.

Também nos diagnósticos das consultas constatou-se uma diminuição das codificações relativas às consultas de vigilância, à exceção da consulta de diabetes, em que até se verificou um aumento no número absoluto de consultas em comparação com anos anteriores. Quanto a outras patologias, realça-se o aumento das consultas com a codificação diagnóstica de patologia psiquiátrica (ansiedade, perturbação do sono e depressão) e infeção urinária, e diminuição da codificação de patologia da coluna vertebral e infeção respiratória superior.

Assim, apesar das limitações encontradas, decorrentes do próprio sistema de informação utilizado e dos vieses associados a uma amostra retrospetiva de mundo real, trata-se da primeira casuística da atividade assistencial de cuidados primários registada durante o primeiro pico da pandemia em Portugal.

\section{REFERÊNCIAS BIBLIOGRÁFICAS}

1. Cennino DJ, Bergman SJ, Olsen KM. Coronavirus disease 2019 (COVID19). Medscape [Internet]; 2020 [updated 2021 Aug 18; cited 2021 Feb 9]. Available from: https://emedicine.medscape.com/article/2500114overview

2. World Health Organization. Coronavirus disease (COVID-19) weekly epidemiological update and weekly operational update [homepage]. WHO; 2020 [updated $2021 \mathrm{Sep}$ 7; cited $2021 \mathrm{Feb} 24$ ]. Available from: https://www.who.int/emergencies/diseases/novel-coronavirus-2019/ situation-reports

3. Berardi C, Antonini M, Genie MG, Cotugno G, Lanteri A, Melia A, et al. The COVID-19 pandemic in Italy: policy and technology impact on health and non-health outcomes. Health Policy Technol. 2020;9(4):45487.

4. Arango C. Lessons learned from the coronavirus health crisis in Madrid, Spain: how COVID-19 has changed our lives in the last 2 weeks. Biol Psychiatry. 2020;88(7):e33-e4.

5. Direção-Geral da Saúde. COVID-19 [homepage]. Lisboa: DGS; 2021 [cited 2021 Feb 24]. Available from: https://covid19.min-saude.pt/

6. Dos Santos CF, Picó-Pérez M, Morgado P. COVID-19 and mental health: what do we know so far? Front Psychiatry. 2020;11:565698.

7. Administração Regional de Saúde do Centro. Reestruturação dos serviços - Em fase (3) de mitigação do COVID-19: orientação n 1/2020 [Internet]. Coimbra: ARS Centro; 2020. Available from: http://www.arscentro.min-saude.pt/wp-content/uploads/sites/6/2020/05/ARSC-1COVID-19-USF-UCSP-13marco20.pdf

8. Administração Regional de Saúde do Centro. Reestruturação dos serviços - Em fase (3) de mitigação do COVID-19 - Unidades funcionais dos ACeS: orientação n 2/2020 [Internet]. Coimbra:ARS Centro; 2020. Available from: http://www.arscentro.min-saude.pt/wp-content/uploads/sites/6/2020/05/ARSC-2-COVID-19-UF-ACeS-ARSC-22marco20.pdf

9. Serviço Nacional de Saúde. Plataforma Transparência [homepage]. Lisboa: Ministério da Saúde; 2021 [cited 2021 Feb 21]. Available from: https://www.sns.gov.pt/transparencia/

10. Rawaf S, Allen LN, Stigler FL, Kringos D, Yamamoto HQ, van Weel C. Lessons on the COVID-19 pandemic, for and by primary care professionals worldwide. Eur J Gen Pract. 2020;26(1):129-33.

11. Public Health Agency of Canada. COVID-19 pandemic guidance for the health care sector [homepage]. Ottawa: PHAC; 2020 [updated 2020 Apr 22; cited 2021 Feb 19]. Available from: https://www.canada.ca/en/ public-health/services/diseases/2019-novel-coronavirus-infection/ health-professionals/covid-19-pandemic-guidance-health-care-sector.html\#a322

12. National Health System England. Guidance and standard operating procedures: general practice in the context of coronavirus (COVID-19), version 4 [Internet]. NHS; 2020 [cited 2021 Feb 19]. Available from: https://www.sheffield-lmc.org.uk/website/IGP217/files/CO485_guidance-and-standard-operating-procedures-general-practice-covid19\%20(1).pdf

13. Park S, Elliott J, Berlin A, Hamer-Hunt J, Haines A. Strengthening the UK primary care response to covid-19. BMJ. 2020;370:m3691.

14. Haldane V, Zhang Z, Abbas RF, Dodd W, Lau LL, Kidd MR, et al. National primary care responses to COVID-19: a rapid review of the litera- 
ture. BMJ Open. 2020;10(12):e041622.

15. Kuitunen I, Ponkilainen VT, Launonen AP, Reito A, Hevonkorpi TP, Paloneva J, et al. The effect of national lockdown due to COVID-19 on emergency department visits. Scand J Trauma Resusc Emerg Med. 2020;28: 114.

16. Escola Nacional de Saúde Pública. Utilização dos serviços de saúde em tempos de Covid-19. Barómetro COVID-19 - Opinião Social [homepage]. Lisboa: ENSP; 2020. Available from: https://barometro-covid19.ensp.unl.pt/utilizacao-dos-servicos-de-saude-em-tempos-de-covid19/

17. Direção-Geral da Saúde. Programa nacional de saúde infantil e juvenil e epidemia de Covid-19: informação nº 008/2020, de 26/03/2020. Lisboa: DGS; 2020.

18. Zhong Y, Clapham HE, Aishworiya R, Chua YX, Mathews J, Ong M, et al. Childhood vaccinations: hidden impact of COVID-19 on children in Singapore. Vaccine. 2021;39(5):780-5.

19. Huston P, Campbell J, Russell G, Goodyear-Smith F, Phillips Jr RL, van Weel C, et al. COVID-19 and primary care in six countries. BJGP Open. 2020;4(4):bjgpopen20X101128.

20. McDonald HI, Tessier E, White JM, Woodruff M, Knowles C, Bates C, et al. Early impact of the coronavirus disease (COVID-19) pandemic and physical distancing measures on routine childhood vaccinations in England, January to April 2020. Euro Surveill. 2020;25(19):2000848.

21. Bramer CA, Kimmins LM, Swanson R, Kuo J, Vranesich P, Jacques-Carroll $L A$, et al. Decline in child vaccination coverage during the COVID19 pandemic: Michigan Care Improvement Registry, May 2016-May 2020. MMWR Morb Mortal Wkly Rep. 2020;69(20):630-1.

22. Jones D, Neal RD, Duffy SR, Scott SE, Whitaker KL, Brain K. Impact of the COVID-19 pandemic on the symptomatic diagnosis of cancer: the view from primary care. Lancet Oncol. 2020;21(6):748-50.

23. Suárez J, Mata E, Guerra A, Jiménez G, Montes M, Arias F, et al. Impact of the COVID-19 pandemic during Spain's state of emergency on the diagnosis of colorectal cancer. J Surg Oncol. 2021;123(1):32-6.

24. Dinmohamed AG, Cellamare M, Visser O, de Munck L, Elferink MA, Westenend PJ, et al. The impact of the temporary suspension of national cancer screening programmes due to the COVID-19 epidemic on the diagnosis of breast and colorectal cancer in the Netherlands. J Hematol Oncol. 2020;13:147.

25. Coma E, Mora N, Méndez L, Benítez M, Hermosilla E, Fàbregas M, et al. Primary care in the time of COVID-19: monitoring the effect of the pandemic and the lockdown measures on 34 quality of care indicators calculated for 288 primary care practices covering about 6 million people in Catalonia. BMC Fam Pract. 2020;21:208.

26. Bhatia RT, Gati S, Papadakis M, Sharma S. The impact of COVID-19 on the continuity of cardiovascular care. Eur Heart J. 2021;42(3):215-7.

27. De Rosa S, Spaccarotella C, Basso C, Calabrò MP, Curcio A, Filardi PP, et al. Reduction of hospitalizations for myocardial infarction in Italy in the COVID-19 era. Eur Heart J. 2020;41(22):2083-8.

28. Mafham MM, Spata E, Goldacre R, Gair D, Curnow P, Bray M, et al. COVID-19 pandemic and admission rates for and management of acute coronary syndromes in England. Lancet. 2020;396(10248):3819.

29. Vieira A, Peixoto VR, Aguiar P, Abrantes A. Rapid estimation of excess mortality during the COVID-19 pandemic in Portugal: beyond report- ed deaths. J Epidemiol Glob Health. 2020;10(3):209-13.

30. Xiang YT, Yang Y, Li W, Zhang L, Zhang Q, Cheung T, et al. Timely mental health care for the 2019 novel coronavirus outbreak is urgently needed. Lancet Psychiatry. 2020;7(3):228-9.

31. Mendes-Santos C, Andersson G, Weiderpass E, Santana R. Mitigating COVID-19 impact on the Portuguese population mental health: the opportunity that lies in digital mental health. Front Public Health. 2020;8:553345.

32. Brooks SK, Webster RK, Smith LE, Woodland L, Wessely S, Greenberg N, et al. The psychological impact of quarantine and how to reduce it: rapid review of the evidence. Lancet. 2020;395(10227):912-20.

33. Passos L, Prazeres F, Teixeira A, Martins C. Impact on mental health due to COVID-19 pandemic: cross-sectional study in Portugal and Brazil. Int J Environ Res Public Health. 2020;17(18):6794.

34. Escola Nacional de Saúde Pública. Como se sentem os Portugueses em tempos de pandemia. Barómetro COVID-19 - Opinião Social [homepage]. Lisboa: ENSP; 2020. Available from: https://barometro-covid19.ensp.unl.pt/como-se-sentem-os-portugueses-em-tempos-de-pandemia/

35. Escola Nacional de Saúde Pública. Barómetro Covid-19: a saúde ocupacional e o risco de Covid-19 [homepage]. Lisboa: ENSP; 2020 Apr 15. Available from: https://www.ensp.unl.pt/a-saude-ocupacional-e-orisco-de-covid-19/

36. Direção-Geral da Saúde. Programa nacional para a saúde mental 2017. Lisboa: DGS; 2017.

37. Rossi R, Socci V, Talevi D, Mensi S, Niolu C, Pacitti F, et al. COVID-19 pandemic and lockdown measures impact on mental health among the general population in Italy. Front Psychiatry. 2020;11:790.

38. Wang C, Pan R, Wan X, Tan Y, Xu L, Ho CS, et al. Immediate psychological responses and associated factors during the initial stage of the 2019 coronavirus disease (COVID-19) epidemic among the general population in China. Int J Environ Res Public Health. 2020;17(5):1729.

39. Qiu J, Shen B, Zhao M, Wang Z, Xie B, Xu Y.A nationwide survey of psychological distress among Chinese people in the COVID-19 epidemic: implications and policy recommendations. Gen Psychiatr. 2020;33(2): e100213.

40. Ammar A, Mueller P, Trabelsi K, Chtourou H, Boukhris O, Masmoudi L, et al. Psychological consequences of COVID-19 home confinement: the ECLB-COVID19 multicenter study. PloS One. 2020;15(11):e0240204.

\section{CONFLITO DE INTERESSES}

Os autores declaram não possuir quaisquer conflitos de interesse.

\section{FINANCIAMENTO}

Este trabalho não recebeu qualquer tipo de suporte financeiro de nenhuma entidade do domínio público ou privado.

\section{ENDEREÇO PARA CORRESPONDÊNCIA}

Pedro Alexandre Vieira

E-mail: pedroafvieira@gmail.com

https://orcid.org/0000-0002-9903-0562

Recebido em 09-02-2021

Aceite para publicação em 01-07-2021 


\section{ABSTRACT}

\section{EFFECTS OF A PANDEMIC IN A PRIMARY CARE UNIT AND ITS POPULATION: A RETROSPECTIVE STUDY}

Objectives: COVID-19 is the first severe pandemic of the modern world, having already collapsed several health systems, forcing them to adapt. This article intends to review the medical activity in a primary care unit during the first pandemic outbreak to assess its impact on the population.

Methods: Retrospective study. Data were retrieved from all medical appointments carried out in a primary care unit between 1 March and 31 July 2020 and in the same period of 2019 and 2018.

Results: There was an increase in the number of medical appointments in 2020 compared to the same period of 2019 and 2018 $(14,871,14,326$, and 12,996 , respectively), the majority being teleconsultations by e-mail or phone in contrast to previous years (71.6\% in $2020,42.6 \%$ in 2019 and $35.2 \%$ in 2018). There was also a decrease in most follow-up and routine appointments (2,562 in 2020, 3,067 in 2019, and 2,975 in 2018) and an increment in psychiatric diagnosis, mainly anxiety-related disorders (362 in 2020, 252 in 2019, and 238 in 2018).

Conclusions: Despite the pandemic, the total number of medical appointments increased in 2020, mostly due to teleconsultations and with a different prevalence of diagnostics. There were fewer follow-up and routine appointments but more diagnoses of psychiatric disorders, probably related to the socio-economic impact of the pandemic. This is the first case series of primary care activity recorded during the first pandemic outbreak in Portugal.

Keywords: COVID-19; Pandemics; Primary health care; Community health centers. 